\title{
Choledochal cysts - an unusual cause of jaundice in adults
}

\author{
Anne-Marie du Plessis, $M B C h B$ \\ Ellie Georgiou, $M B C h B$ \\ Savvas Andronikou, MB BCh, FCRad, FRCR, PhD \\ Departments of Radiology and Surgery, Stellenbosch University, \\ Tygerberg Hospital, Tygerberg
}

\begin{abstract}
Objectives: This is a good example with interesting imaging of a condition which rarely presents in adulthood.

Methodology: Case was described and a review and short summary of the literature was done.

Conclusions: The complete resection of choledochal cysts is mandatory because of risk of malignant transformation.
\end{abstract}

Choledochal cysts occur in approximately 1:10 000 to $1: 150000 .{ }^{1}$ Of these only $20-30 \%$ are diagnosed in adults, ${ }^{2}$ with $80 \%$ of cases diagnosed reported as being of the type I variety. Complete surgical excision with biliary reconstruction is considered the treatment of choice rather than biliary enteric bypass procedures. This minimises the known risk of malignancy and the development of recurrent cholangitis or pancreatitis that may occur with these cystic lesions. ${ }^{1-3}$

The diagnosis, surgical findings and treatment of an adult patient who presented to us with a type I choledochal cyst are described and the epidemiology, diagnosis, treatment and cancer risk of choledochal cysts are discussed.

\section{Case report}

A 33-year-old female presented with complaints of a right upper quadrant mass increasing in size over a 6-month period. She gave no history of melaena or haematemesis and had no gastrointestinal complaints but had noticed her eyes becoming progressively yellow.

On examination she was found to be jaundiced with a large mass occupying the right side of her abdomen, thought to be separate from the liver. She had a palpable spleen, no ascites and all other systems were found to be normal.

Liver enzymes were all marginally raised, hepatitis studies, echinococcus and amoebiasis serology were all negative.

Ultrasound showed a large cystic mass thought of be part of the biliary tree and a computed tomography (CT) scan was requested which confirmed a cystic mass (HU 5) at the porta hepatic (Figs $1 \& 2$ ). Differential diagnosis included enteric duplication cyst, lymphoma and especially in an African setting, hydatid cyst and amoebic abscess.

On surgical exploration a large bile-containing cyst was found, arising from the proximal aspect of the common bile duct, compatable with a type I choledochal cyst. Complete excision of the cyst was performed and a choledochoduodenostomy was done.

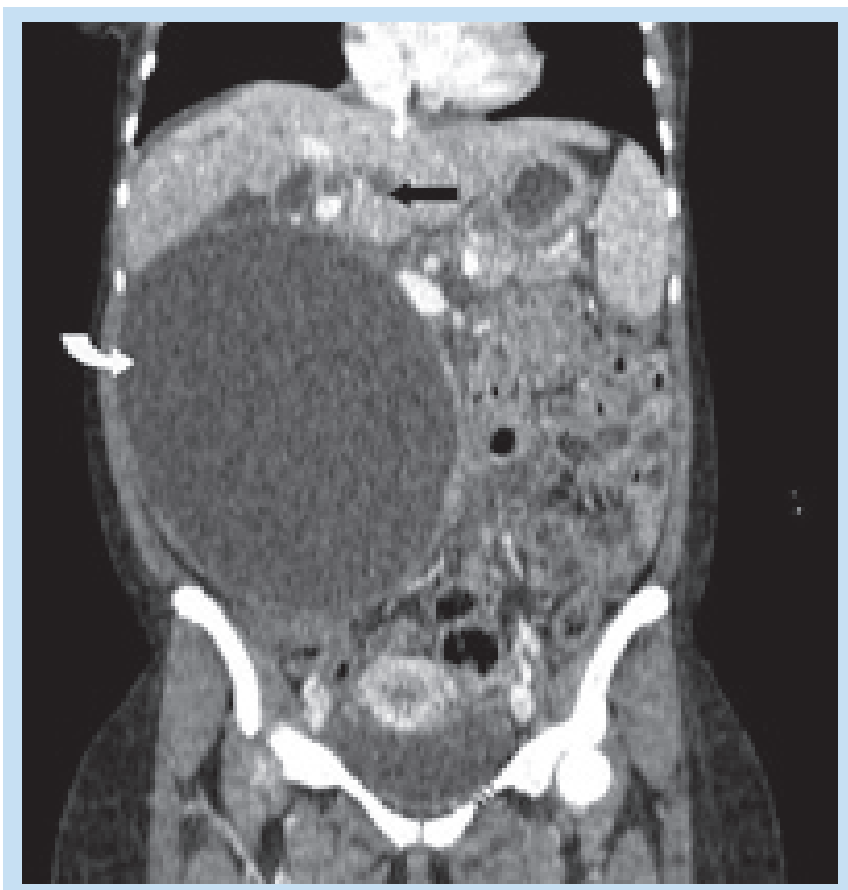

Fig. 1. Coronal reconstruction of multislice CT abdomen demonstrates a massive cystic lesion (curved arrow) at the right inferior border of the liver, choledochal cyst, with associated intrahepatic biliary dilatation (black arrow).

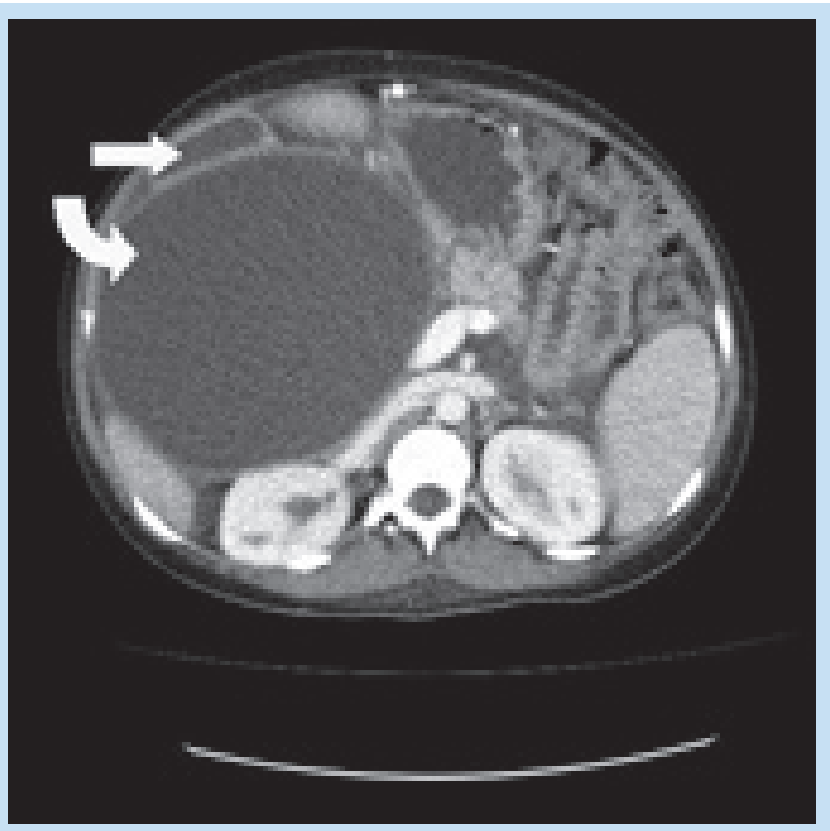

Fig. 2. Axial CT abdomen demonstrates the choledochal cyst located in the right hemi-abdomen (curved arrow) The gall bladder is indicated with a straight arrow. 


\section{Discussion}

Choledochal cysts are classified according to the Todani modification of the original Alonso-Ley classification. ${ }^{1,2}$ Six groups are identified.

Type I is a fusiform dilatation of the extra hepatic bile duct, as was found in our patient. Type II is a single extrahepatic diverticulum. Type III is a dilatation of the intraduodenal portion of the bile duct. Type IV consists of two subtypes: IVa which is combined intra- and extrahepatic dilatation and IVb which is multiple extrahepatic cysts only. Type $\mathrm{V}$ is also known as Caroli's disease and consists of cystic dilatation of the intrahepatic biliary system. It is also associated with hepatic fibrosis. ${ }^{2-4}$

The aetiology of choledochal cysts remains unproven but an anomalous pancreaticobiliary junction has been reported in choledochal cyst disease. Babbit ${ }^{3}$ proposed a theory that suggests a high insertion of the bile duct into the pancreatic duct allowing pancreatic enzymes to reflux into the bile duct. This causes inflammation, weakening and fibrosis of the bile duct and the distal obstruction leads to progressive dilatation of the biliary tree. ${ }^{2,3}$

Children present with the classic triad of abdominal mass, abdominal pain and jaundice. Adults however present mainly with pain ${ }^{2,3}$ and complications of longstanding cysts such as recurrent cholangitis, pancreatitis and malignancies. ${ }^{4}$ A palpable mass is rare as a presenting feature in adults.

Laboratory tests have not been proven to be useful in establishing a diagnosis and it has been shown that hepatic transaminase, bilirubin and white cell count were normal in up to $50 \%$ of patients at time of diagnosis. ${ }^{2}$

Imaging however is essential in making a diagnosis. Ultrasound and CT are useful for detecting cysts, but differentiation from other fluid collections and confirmation of the biliary origin of the cyst can sometimes be difficult. Direct contrast cholangiography is used to define extent of involvement and visualise the remaining biliary tree. ${ }^{2,45}$ Contrast cholangiography is invasive though, and magnetic resonance cholangiography (MRCP) is a non-invasive technique, shown by Govil et al. ${ }^{6}$ to be as accurate in confirming the diagnosis of choledochal cysts and defining extent of involvement pre-operatively.
There is an increased risk of malignancy in choledochal cysts and the occurrence of cancer is most marked in adults. Type I has the highest predominance of cancer, followed by the type IV cyst. The type III cyst is the least common type harbouring cancer. Most of the tumours found are adenocarcinomas, although squamous and anaplastic cell carcinomas are occasionally discovered. The most common site of occurrence is the posterior cyst wall. Prognosis is poor and most patients die within 2 years of diagnosis. ${ }^{1}$

Early surgical intervention protocols consisted of drainage and enterostomy. ${ }^{2,3}$ Complications occurring with this approach included anastomotic strictures, calculi associated with stasis, recurrent cholangitis and most importantly, malignant disease. Currently total excision with reconstuction of the biliary tree by means of hepaticojejenostomy is considered to be the treatment of choice although type III cysts have been shown to respond adequately when managed with endoscopic sphincterotomy. ${ }^{3}$ This approach reduces the risk of malignant disease by $60-70 \%{ }^{3}$

\section{Conclusion}

In conclusion, choledochal cysts in adults are rare and usually present with complications of longstanding cysts. Diagnosis is made by ultrasound, CT contrast cholangiography and MRCP. Total cyst excision is performed to minimise malignant transformation and prevent complications of pancreatitis and recurrent cholangitis.

1. Weyant MJ, Maluccio MA, Bertagnolli MM, et al. Choledochal cysts in adults: a report of two cases and review of the literature. Am J Gastroenterol 1998; 93: 2580-2583.

2. Lopez RR, Pinson CW, Campell JR, et al. Variation in management based on type of choledochal cyst. Am J Surg 1991; 161: 612-615.

3. Scudamore CH, Hemming AW, Teare JP, et al. Surgical management of choledochal cysts. Am J Surg 1994, 167: 479-500.

4. Jesudason SRB, Govil S, Mathai V, et al. Choledochal cysts in adults. Ann R Coll Surg Engl 1997; 79; 410413

5. Lipsett PA, Pitt HA, Colombani PM, et al. Choledochal cyst disease: a changing pattern of presentation Ann Surg 1994; 220: 644-652.

6. Govil S, Justus A, Korah I, et al. Choledochal cysts: evaluation with MR cholangiography. Abdom Imaging $1998 ; 23: 616-619$ 